\title{
The Atopic Dermatitis Control Tool: A High-Performance Tool for Optimal Support
}

\author{
Delphine STAUMONT-SALLÉ ${ }^{1,2}$, Charles TAIEB ${ }^{3}$, Stephanie MERHAND ${ }^{4}$ and Jason SHOURICK ${ }^{5}$ \\ ${ }^{1}$ Department of Dermatology, Centre Hospitalier Universitaire (CHU) de Lille, ${ }^{2}$ INSERM U995, Lille Inflammation Research International Center, \\ Lille, ${ }^{3}$ Patients Priority Department, European Market Maintenance Assessment (EMMA), Fontenay Sous-Bois, ${ }^{4}$ French Eczema Association, \\ Redon, and ${ }^{5}$ Dermatologist, Epidemiologist, Hôpital de Toulouse, Toulouse, France
}

\begin{abstract}
The evaluation of global atopic dermatitis control is key to minimizing disease burden. The Atopic Dermatitis Control Tool (ADCT) has been developed for this purpose. Participants (diagnosed by a physician) were recruited to this observational cross-sectional study using real-life methodology and completed a questionnaire on sociodemographic and personal information. The ADCT algorithm, described by Pariser, was used to categorize patients as having controlled or uncontrolled atopic dermatitis. Data were collected for 1,606 patients. Median age of the patients was 40 years, and 1,023 (63.7\%) patients were women. A total of $1,146(71.4 \%)$ patients had uncontrolled atopic dermatitis according to the ADCT score. Patients with uncontrolled disease were at significantly higher risk of a high stress level and were more likely to be absent from work than those with controlled disease. In conclusion, a key factor for predicting disease burden in atopic dermatitis is patient self-assessed disease control in terms of multiple dimensions: stress, sleep, quality of life, work absenteeism and loss of productivity.
\end{abstract}

Key words: atopic dermatitis; Atopic Dermatitis Control Tool; patient centricity.

Accepted Nov 29, 2021; Epub ahead of print Nov 29, 2021

Acta Derm Venereol 2021; 101: adv00618.

Corr: Charles Taieb, Patient Priority Department, European Market Maintenance Assessment, FR-94120 Fontenay sous Bois, France. E-mail: charles.taieb@emma.clinic

A topic dermatitis (AD) is an inflammatory skin disease that affects $1.2-9.7 \%$ of the adult population (1-4). Results from the "Objectif Peau" project in France, which was initiated by the French Society of Dermatology, showed a $4.65 \%$ prevalence of AD (5). AD is characterized by swelling, skin pain, and itching, and is associated with a high disease burden (6-9). AD has been shown to significantly impact sleep quality, one of the dimensions of disease burden $(10,11)$. Psychological stress and $\mathrm{AD}$ create a vicious cycle: stress induces flares, and flares induce psychological stress $(12,13)$. $\mathrm{AD}$ also significantly impacts work absenteeism, as well as causing a decline in productivity (14-17). Therefore, the clinical evaluation of $\mathrm{AD}$ is difficult, since multiple symptoms and burden dimensions must be taken into account. Evaluating global disease control and, more

\section{SIGNIFICANCE}

Assessment of overall disease control reported by patients during clinical encounters is essential to ensure a minimal disease burden. In a context in which healthcare professionals are overburdened, leading to sparse follow-up consultations with patients, the self-reported nature of the Atopic Dermatitis Control Tool is useful, as patients can assess the control of their disease before consulting their healthcare provider. This study shows that the use of this tool in a large population is relevant.

specifically, patient self-reported global disease control, during clinical encounters, is key to minimizing disease burden. However, until recently, a holistic scale has not been available to implement self-reported disease control. The Atopic Dermatitis Control Tool (ADCT) was developed for this purpose $(18,19)$. The tool includes 6 questions that evaluate the overall severity of symptoms, days with intense episodes of itching, bothersome intensity, problems with sleep, impact on daily activities, and impact on mood or emotions during the preceding week. A short completion time ( $<2 \mathrm{~min})$ makes the ADCT easy to use during consultation, especially as an alert for loss of disease control. Furthermore, an algorithm has been developed to easily separate patients with controlled disease from those with uncontrolled disease. The Dermatology Life Quality Index (DLQI) has been used to demonstrate that ADCT is highly correlated with quality of life (QoL), but only moderately correlated with productivity and activity impairment. However, the role of disease control in all these elements is not always clear. The aim of this study was to evaluate the effect of uncontrolled AD on stress, sleep quality, work absenteeism and loss of productivity.

\section{MATERIALS AND METHODS}

\section{Study design}

This was an observational, cross-sectional study. The study was approved by local ethics committees at CHU of Brest, France (reference number 2020-A02110-39).

\section{Study population}

Survey participants were recruited between January and February 2021, either through the national AD patient association (Associa- 
tion Française de l'eczéma) or from a representative sample of French adults. The national association posted an announcement and link on their website and in their newsletter, as well on social networks. The representative sample was recruited by a polling institute (HC Conseil Paris, France) between January and February 2021 from the general adult population above 18 years of age using stratified, proportional sampling with a replacement design. Respondents who reported being diagnosed with AD by a physician were invited to participate in the study. The inclusion criteria were: ability to understand French; provision of consent to participate in the study after receipt of written information about the study; age above 18 years.

\section{Data collection}

Respondents answered a questionnaire regarding sociodemographic and personal information. Questions on age, sex, professional level, history of $\mathrm{AD}$ and treatment were included.

AD control was assessed by the ADCT. The ADCT algorithm described in Pariser et al. (19) was used to categorize patients as having controlled or uncontrolled AD.

Quality of life was assessed using the DLQI and the 12-item Short-Form Survey (SF-12). The DLQI is a scoring system (20). A DLQI score between 6 and 10 indicates a moderate effect on the patient's life, a score between 11 and 20 indicateds a significant effect on the patient's life, and a score between 21 and 30 indicates an extremely significant effect on the patient's life (21). Stress was assessed using the Perceived Stress Scale questionnaire (22). A score lower than 21 indicates low stress, between 21 and 26 indicates moderate stress, and above 26 indicates high stress (23). Patients were asked if they had missed work through sick leave, paid annual leave or unpaid leave during the previous year. Patient loss of productivity for a relevant activity was evaluated by the question "Do you feel you were less productive at work or school during the last 3 months as a result of your skin disease?" using a Likert scale (Very Often, Often, Sometimes, Rarely, and Never).

Clinical severity was assessed with the Patient-Oriented Eczema Measure (POEM) (24). This self-assessed measurement tool is used to monitor disease activity in adults with atopic eczema. Questions are included on the frequency of occurrence of 7 symptoms during the preceding week (itching, sleep, bleeding, weeping, skin cracking, skin flaking off, and skin dryness) and scored from 0 to 28 . There are 3 categories of scores: mild (0-7), moderate (8-16), and severe (17-28) (25).

\section{Statistical analyses}

Categorical values are presented as numbers and percentages, and continuous variables are presented in terms of first and third median quartiles. The ADCT was used to categorize patients with controlled and uncontrolled disease. Categorical variables were compared using the $\chi^{2}$ test, and continuous variables were compared using the $t$-test. An assessment was performed to determine whether the ADCT score was subject to confusion bias and to compare disease control, as categorized by ADCT, with long-term severity based on the POEM score. Logistic regression was performed with the following outcomes: $(i)$ high stress, as defined by the PSS; (ii) an extremely or very large effect on QoL, as defined by the DLQI; (iii) work absenteeism, defined as patients declaring they had missed work at least once during the preceding year; and (iv) loss of productivity, defined as a patient response of "often" or "very often" to the question on productivity in a relevant activity. The explanatory variables used in all the investigated models were sex, age, disease control (according to the ADCT score), severity (according to the POEM score), current topical treatment and current systemic treatment.
Table I. Comparison of controlled and not controlled patients

\begin{tabular}{lcll}
\hline & $\begin{array}{l}\text { Controlled AD } \\
n=460 \\
n(\%)\end{array}$ & $\begin{array}{l}\text { Not controlled AD } \\
n=1,146 \\
n(\%)\end{array}$ & $p$-value \\
Variable & $37(8)$ & $122(10.6)$ & $<0.001$ \\
\hline Age & $74(16.1)$ & $321(28)$ & \\
$18-24$ years & $101(22)$ & $301(26.3)$ & \\
$25-34$ years & $91(19.8)$ & $194(16.9)$ & \\
$35-44$ years & $80(17.4)$ & $125(10.9)$ & \\
$45-54$ years & $77(16.7)$ & $83(7.2)$ & \\
$55-64$ years & & & \\
$>65$ years & $309(67.2)$ & $714(62.3)$ & \\
Sex & $151(32.8)$ & $432(37.7)$ & \\
Women & & & \\
Men & $394(85.7)$ & $379(33.1)$ & \\
Severity (POEM) & $64(13.9)$ & $607(53)$ & $<0.001$ \\
Mild & $2(0.4)$ & $160(14)$ & $<0.001$ \\
Moderate & & & \\
Severe & $48(10.4)$ & $235(20.5)$ & $<0.001$ \\
Treatment & $266(57.8)$ & $803(70.1)$ & \\
Unsatisfied of treatment & $32(7)$ & $229(20)$ & \\
Topical treatment & &
\end{tabular}

AD: atopic dermatitis; POEM: Patient Oriented Eczema Measure.

\section{RESULTS}

Data were collected on 1,606 patients. Median age of the patients was 40 years, and 1,023 (63.7\%) patients were women. AD was not controlled in $1,146(71.4 \%)$ patients according to the ADCT score. Table $\mathbf{I}$ is a comparison of data on patients with controlled and uncontrolled disease.

\section{Severity}

According to the POEM scores of the respondents, 773 (48.1\%) presented mild AD, $671(41.8 \%)$ moderate $\mathrm{AD}$, and $162(10.1 \%)$ severe $\mathrm{AD}$. AD was controlled in significantly fewer patients with severe disease than patients with moderate and mild AD $(p<0.001): 379$ (49\%) patients presented uncontrolled mild AD, 607 (90.5\%) uncontrolled moderate AD, and 160 (98.8\%) uncontrolled severe AD. Compared with patients with controlled $\mathrm{AD}$, those with uncontrolled $\mathrm{AD}$ were at higher risk of feeling that their disease was worsening (346 (30.2\%) patients with uncontrolled AD vs $32(7 \%)$ patients with controlled $\mathrm{AD}, p<0.001)$ and of being dissatisfied with their treatment $(235$ (20.5\%) patients with uncontrolled AD vs $48(10.4 \%)$ patients with controlled $\mathrm{AD}, p<0.001)$ ).

Table II. Sleep issues

\begin{tabular}{lcll}
\hline & $\begin{array}{l}\text { Controlled AD } \\
n=460 \\
n(\%)\end{array}$ & $\begin{array}{l}\text { Not controlled } \\
\text { AD } n=1,146 \\
n(\%)\end{array}$ & $p$-value \\
\hline Variable & $24(5.2)$ & $586(51.1)$ & $<0.001$ \\
\hline Difficulty falling asleep due to AD & $18(3.9)$ & $626(54.6)$ & $<0.001$ \\
Awakens at night due to AD & & & $<0.001$ \\
Wakes up in the morning & $72(15.7)$ & $63(5.5)$ & \\
$\quad$ Well rested & $176(38.3)$ & $373(32.5)$ & \\
Rested & $181(39.3)$ & $562(49)$ & \\
A bit tired & $31(6.7)$ & $148(12.9)$ & \\
Really tired & &
\end{tabular}

AD: atopic dermatitis. 


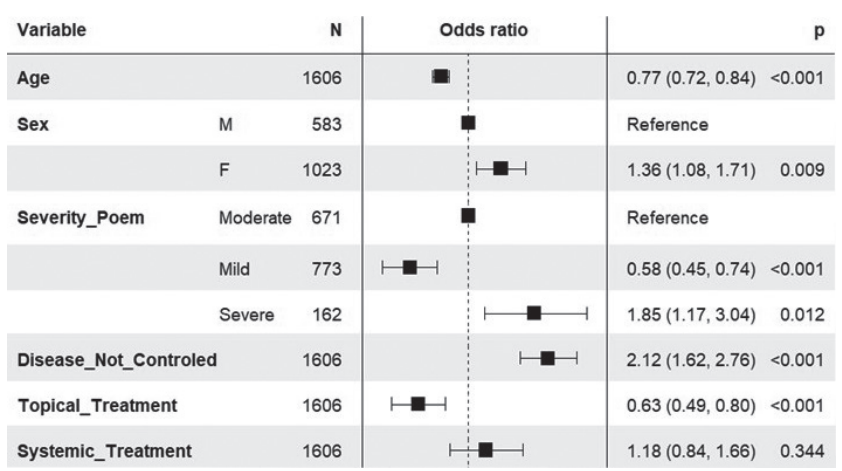

Fig. 1. Forest plot for a multivariate logistic regression analysis with high perceived stress as the outcome (defined as a Perceived Stress Scale score above 26). Uncontrolled disease is defined using the atopic dermatitis control tool algorithm.

\section{Sleep issues}

Compared with patients with controlled AD, those with uncontrolled $\mathrm{AD}$ were at a higher risk of feeling extremely tired (148 (12.9\%) with uncontrolled AD vs 31 $(6.7 \%)$ with controlled AD, $p<0.001)$. Compared with patients with controlled $\mathrm{AD}$, those with uncontrolled $\mathrm{AD}$ had more difficulty falling asleep due to $\mathrm{AD}$ (586 (51.1\%) with uncontrolled $\mathrm{AD}$ vs 24 (5.2\%) with controlled $\mathrm{AD}$, $p<0.001)$ and were more at risk of waking up during the night due to $\mathrm{AD}(626(54.6 \%)$ patients with uncontrolled $\mathrm{AD}$ vs 18 (3.9\%) patients with controlled $\mathrm{AD}, p<0.001)$. Compared with patients with controlled AD, those with uncontrolled AD were at higher risk of taking more than $20 \mathrm{~min}$ to fall asleep, sleeping less than $6 \mathrm{~h}$ more than 3 times per week and waking up during the night with difficulty falling back asleep (Table II).

\section{Stress (Perceived Stress Scale)}

Based on the PSS scores of the respondents, only 221 (13.8\%) presented a low stress level, $329(20.5 \%)$ presented a medium stress level and 1,056 (65.8\%) presented a high stress level. Compared with patients with controlled $\mathrm{AD}$, patients with uncontrolled disease showed a significantly higher risk of having a high stress level $(840$ $(73.3 \%)$ for patients with uncontrolled AD vs 216 (47\%) for patients with controlled $\mathrm{AD}, p<0.001)$. Multivariate

Table III. Feeling, patient's impact

\begin{tabular}{lcll}
\hline & $\begin{array}{l}\text { Controlled } \\
\text { VD } n=460\end{array}$ & Not controlled \\
Variable & $n(\%)$ & $n(\%)$ & $p$-value \\
\hline DLQI & & & $<\mathbf{0 . 0 0 1}$ \\
$\quad$ No effect on patient's life & $260(56.5)$ & $115(10)$ & \\
$\quad$ Small effect on patient's life & $167(36.3)$ & $324(28.3)$ & \\
Moderate effect on patient's life & $21(4.6)$ & $245(21.4)$ & \\
$\quad$ Very large effect on patient's life & $7(1.5)$ & $390(34)$ & \\
$\quad$ Extremely large effect on patient's life & $5(1.1)$ & $72(6.3)$ & $<0.001$ \\
Perceived stress & & & \\
Low & $113(24.6)$ & $108(9.4)$ & \\
Medium & $131(28.5)$ & $198(17.3)$ & \\
High & $216(47)$ & $840(73.3)$ & \\
\hline
\end{tabular}

AD: atopic dermatitis; DLQI: Dermatology Life Quality Index.

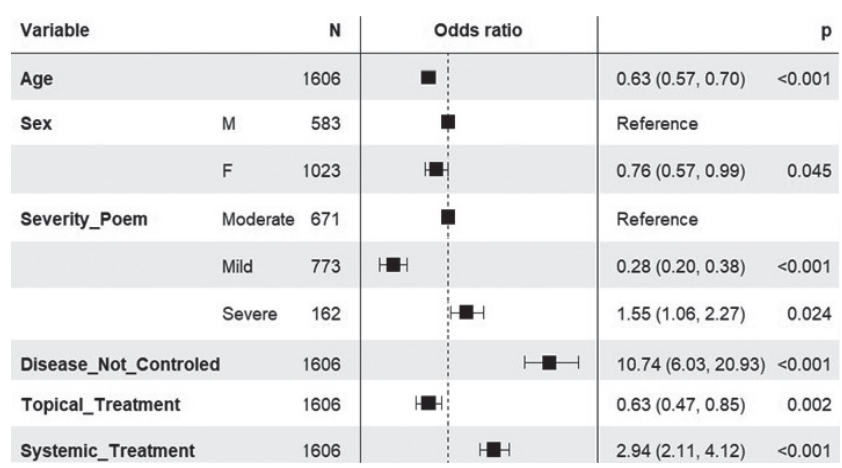

Fig. 2. Forest plot for a multivariate logistic regression analysis with a very or an extremely large effect on quality of life as the outcome (defined as a Dermatology Life Quality Index score above 10). Uncontrolled disease is defined using the atopic dermatitis control tool algorithm.

analysis (Fig. 1) with high stress as the outcome showed an odds ratio (OR) of 2.12 (95\% confidence interval (95\% CI) $1.62,2.76), p<0.001$ for patients with uncontrolled disease (Table III).

\section{Quality of life}

Based on the DLQI scores of patients, the effect of AD on QoL was reported as non-existent by 375 (23.3\%) patients, small by 491 (30.6\%) patients, moderate by 266 (16.6\%) patients, very large by 397 (24.7\%) patients, and extremely large by 77 (4.8\%) patients. Compared with patients with controlled $\mathrm{AD}$, patients with uncontrolled disease were more at risk of perceiving $\mathrm{AD}$ as having a very or extremely large effect on their QoL (462 (40.3\%) patients with uncontrolled AD vs $12(2.6 \%)$ patients with controlled AD, $p<0.001)$ ). However, the DLQI scores for patients with uncontrolled $\mathrm{AD}$ showed that $115(10 \%)$ patients reported AD had no effect on their QoL, and $324(28.3 \%)$ patients reported AD had a small effect on their QoL. Multivariate analysis (Fig. 2) with very large or extremely large deterioration of QoL as the outcome showed an OR of 10.7 (95\% CI 6.03, 20.9), $p<0.001$ for patients with uncontrolled disease (Table III).

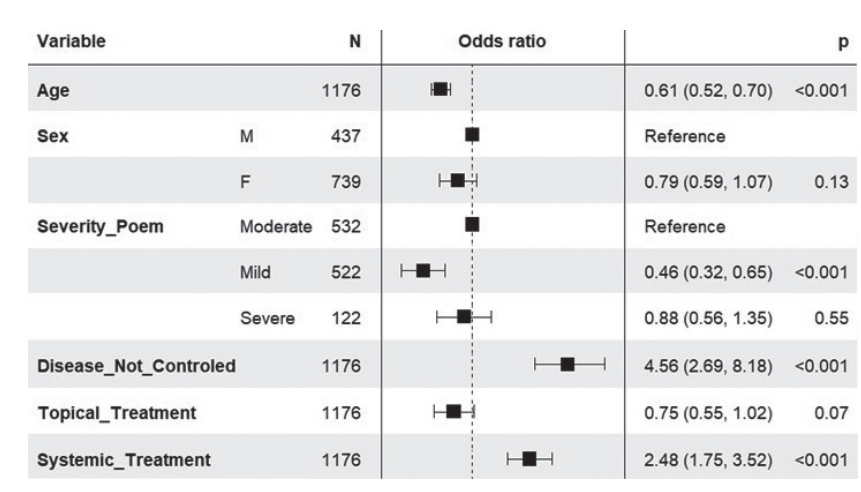

Fig. 3. Forest plot for a multivariate logistic regression analysis with work absenteeism during the preceding year as the outcome (defined as missing at least 1 day of work because of atopic dermatitis). Uncontrolled disease is defined using the atopic dermatitis control tool algorithm. 
Table IV. Repercussion on professional activity: absenteeism and loss of production

\begin{tabular}{llll}
\hline Variable & $\begin{array}{l}\text { Controlled AD } \\
n=460 \\
n(\%)\end{array}$ & $\begin{array}{l}\text { Not controlled AD } \\
n=1,146 \\
n(\%)\end{array}$ & $p$ \\
\hline $\begin{array}{l}\text { Presenteeism } \\
\text { Very often }\end{array}$ & $4(1.1)$ & $56(5.5)$ & $<0.001$ \\
Often & $9(2.5)$ & $121(11.8)$ & \\
Sometimes & $35(9.6)$ & $291(28.3)$ & \\
Rarely & $47(12.9)$ & $257(25)$ & \\
$\quad$ Never & $270(74)$ & $302(29.4)$ & $<0.001$ \\
Absenteeism & $10(3.4)$ & $215(24.5)$ & $<0.001$ \\
Sick leave & $11(3.7)$ & $178(20.3)$ & $<0.001$ \\
$\quad \begin{array}{l}\text { Paid annual leave } \\
\text { Unpaid leave }\end{array}$ & $9(3)$ & $155(17.7)$ & \\
\hline
\end{tabular}

AD: atopic dermatitis.

\section{Work absenteeism}

A total of $315(26.8 \%)$ of the patients missed work at least once during the preceding year due to AD. Among these patients, $225(19.1 \%)$ missed work with sick leave, 189 (16.1\%) with paid annual leave and $164(13.9 \%)$ with unpaid leave. The median number of missed days of work among patients who missed at least 1 day of work was 29 quartiles $(95 \%$ CI 17, 42). Compared with patients with controlled disease, patients with uncontrolled disease had more opportunities to miss work (298 (33.9\%) patients with controlled disease vs $17(5.7 \%)$ patients with controlled disease, $p<0.001)$. Multivariate analysis (Fig. 3) with missing work at least once during the preceding year as the outcome showed an OR of 4.56 (95\% CI 2.69, 8.18), $p<0.001$, for patients with uncontrolled disease (Table IV).

\section{Loss of productivity}

Regarding the frequency of being less efficient due to AD, $60(4.3 \%)$ patients answered "very often", $130(9.3 \%)$ answered "often", 326 (23.4\%) answered "sometimes", $304(21.8 \%)$ answered "rarely" and 572 (41.1\%) answered "never". Compared with patients with controlled disease, patients with uncontrolled disease were more at risk of being less efficient often or very often (177 (15.4\%) patients

\begin{tabular}{|c|c|c|c|c|c|c|}
\hline \multirow{2}{*}{$\begin{array}{l}\text { Variable } \\
\text { Age }\end{array}$} & & \multirow{2}{*}{$\begin{array}{r}\mathbf{N} \\
1606\end{array}$} & \multicolumn{2}{|c|}{ Odds ratio } & \multicolumn{2}{|r|}{ p } \\
\hline & & & 대 & & $0.70(0.61,0.80)$ & $<0.001$ \\
\hline \multirow[t]{2}{*}{ Sex } & M & 583 & 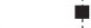 & & Reference & \\
\hline & $\mathrm{F}$ & 1023 & $1=$ & & $0.93(0.66,1.30)$ & 0.661 \\
\hline \multirow[t]{3}{*}{ Severity_Poem } & Moderate & 671 & $\boldsymbol{\varphi}$ & & Reference & \\
\hline & Mild & 773 & 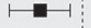 & & $0.55(0.36,0.84)$ & 0.006 \\
\hline & Severe & 162 & & $\longmapsto-1$ & $2.53(1.67,3.83)$ & $<0.001$ \\
\hline Disease_Not_Controled & & 1606 & & $\longmapsto-1$ & $2.93(1.60,5.75)$ & $<0.001$ \\
\hline Topical_Treatment & & 1606 & $\mapsto$ & & $0.67(0.47,0.95)$ & 0.023 \\
\hline Systemic_Treatment & & 1606 & & $\mapsto-1$ & $2.11(1.45,3.03)$ & $<0.001$ \\
\hline
\end{tabular}

Fig. 4. Forest plot for a multivariate logistic regression analysis with being less efficient at work often or very often as the outcome. ("Do you feel you were less productive at work or school during the last 3 months as a result of your skin disease?"). Uncontrolled disease is defined using the atopic dermatitis control tool algorithm. with controlled disease vs $13(2.8 \%)$ patients with controlled disease, $p<0.001)$ ). Multivariate analysis (Fig. 4) with being less efficient often or very often, as the outcome showed an OR of 2.93 (95\% CI 1.6, 5.75), $p<0.001$ for patients with uncontrolled disease (Table IV).

\section{DISCUSSION}

This study shows that self-assessed disease control is a key factor in predicting disease burden in terms of multiple dimensions: stress, sleep, health-related QoL, work absenteeism, and loss of productivity. The study showed that perceived control, based on the ADCT, is better at predicting burden than symptom severity assessment by the POEM scale. The role of ADCT in clinical practice was clarified: although the majority $(71.4 \%)$ of respondents were categorized as having uncontrolled disease, $38.3 \%$ of the patients reported no or small differences in QoL, and $26.7 \%$ presented low or medium stress levels. This result indicates the difference between a global short-score evaluation of disease control and a more thorough investigation of physical and psychological dimensions of the disease (26). Therefore, we conclude that the ADCT is a very good tool for the early detection of loss of disease control in patients in whom a low symptom level has already been achieved. Considering the low threshold used to define uncontrolled disease in the ADCT, we recommend the ADCT is not used as an initial evaluation tool, but as a follow-up tool. The ADCT is especially useful for disease self-monitoring in following up patients with $\mathrm{AD}$ receiving systemic treatment with reduced symptoms (27).

In contexts in which health professionals are overwhelmed with work, the spacing between patient follow-up consultations increases (28). The self-reported nature of the ADCT increases the utility of this tool, because patients can evaluate their own disease control prior to consulting their physician. The ADCT could also be used by doctors and patients as a monitoring tool. The patient could be asked to complete the questionnaire, and a health professional would be alerted in the event of uncontrolled AD. The health professional could then invite the patient for a consultation. An equivalent system exists for some diseases for which monitoring is necessary, such as heart failure, in which uncontrolled weight is a warning sign. In the event of rapid weight increase, a connected scale is used to alert a health professional, who then contacts the patient to take action (29).

However, the ADCT should not be used as the sole measure of a patient's AD state and should be completed in conjunction with subjective measures of QoL (DLQI), as well as objective measures of severity and activity and, when needed, specific patient-reported outcomes for dimensions, such as stress or sleep quality.

This study has some limitations, the most prominent of which is that a precise treatment and disease history 
was not obtained from the respondents, which would have enabled the target population to be better defined.

In conclusion, this study demonstrated the high predictive value of ADCT for disease burden and verified the ADCT as being a very good tool for the early detection of loss of disease control in patients in whom a low symptom level has been achieved.

\section{ACKNOWLEDGEMENTS}

The authors would like to thank and dedicate this study to Gaëtan Fuzet, who passed away in his $29^{\text {th }}$ year on 28 March 2021 . He supported and made a large contribution to this study.

This study received funding from SANOFI.

The authors have no conflicts of interest to declare.

\section{REFERENCES}

1. Brunner PM, Silverberg JI, Guttman-Yassky E, Paller AS, Kabashima K, Amagai M, et al. Increasing comorbidities suggest that atopic dermatitis is a systemic disorder. J Invest Dermatol 2017; 137: 18-25.

2. Thijs JL, Strickland I, Bruijnzeel-Koomen CAFM, Nierkens S, Giovannone B, Knol EF, et al. Serum biomarker profiles suggest that atopic dermatitis is a systemic disease. J Allergy Clin Immunol 2018; 141: 1523-1526.

3. Oliveira C, Torres T. More than skin deep: the systemic nature of atopic dermatitis. Eur J Dermatol EJD 2019; 29: 250-258.

4. Bylund S, Kobyletzki L, Svalstedt M, Svensson. Prevalence and incidence of atopic dermatitis: a systematic review. Acta Derm Venereol 2020; 100: adv00160.

5. Richard M-A, Corgibet F, Beylot-Barry M, Barbaud A, Bodemer C, Chaussade V, et al. Sex- and age-adjusted prevalence estimates of five chronic inflammatory skin diseases in France: results of the « OBJECTIFS PEAU » study. J Eur Acad Dermatol Venereol 2018; 32: 1967-1971.

6. Langan SM, Irvine AD, Weidinger S. Atopic dermatitis. The Lancet 2020; 396: 345-360.

7. Koszorú K, Borza J, Gulácsi L, Sárdy M. Quality of life in patients with atopic dermatitis. Cutis 2019; 104: 174-177.

8. Kage P, Simon J, Treudler R. Atopic dermatitis and psychosocial comorbidities. J Dtsch Dermatol Ges 2020; 18: 93-102.

9. Girolomoni G, Gadkari A, Auziere S, Puig L, Barbarot S, Chosidow $\mathrm{O}$, et al. The patient-reported disease burden in adults with atopic dermatitis: a cross-sectional study in Canada and Europe. Value Health 2017; 20: A807.

10. Chang Y-S, Chiang B-L. Sleep disorders and atopic dermatitis: a 2-way street? J Allergy Clin Immunol 2018; 142: 1033-1040.

11. Mann C, Dreher M, Weeß H-G, Staubach P. Sleep disturbance in patients with urticaria and atopic dermatitis: an underestimated burden. Acta Derm Venereol 2020; 100: adv00073.

12. Lin T-K, Zhong L, Santiago JL. Association between stress and the HPA axis in the atopic dermatitis. Int J Mol Sci 2017; 18: 2131.

13. Suárez AL, Feramisco JD, Koo J, Steinhoff M. Psychoneuroim- munology of psychological stress and atopic dermatitis: pathophysiologic and therapeutic updates. Acta Derm Venereol 2012; 92: 7-15.

14. Chung J, Simpson EL. The socioeconomics of atopic dermatitis. Ann Allergy Asthma Immunol Off Publ Am Coll Allergy Asthma Immunol 2019; 122: 360-366.

15. Ariëns LFM, van Nimwegen KJM, Shams M, de Bruin DT, van der Schaft J, van Os-Medendorp $\mathrm{H}$, et al. Economic burden of adult patients with moderate to severe atopic dermatitis indicated for systemic treatment. Acta Derm Venereol 2019; 99: 762-768.

16. Stróżek J, Samoliński BK, Kłak A, Gawińska-Drużba E, Izdebski R, Krzych-Fałta $E$, et al. The indirect costs of allergic diseases. Int J Occup Med Environ Health 2019; 32: 281-290.

17. Le PH, Vo TQ. Economic burden and productivity loss related to eczema: a prevalence-based follow-up study in Vietnam. JPMA J Pak Med Assoc 2019; 69: S57-S63.

18. Simpson E, Eckert L, Gadkari A, Mallya UG, Yang M, Nelson $L$, et al. Validation of the Atopic Dermatitis Control Tool (ADCT C) using a longitudinal survey of biologic-treated patients with atopic dermatitis. BMC Dermatol 2019; 19: 15.

19. Pariser DM, Simpson EL, Gadkari A, Bieber T, Margolis DJ, Brown $M$, et al. Evaluating patient-perceived control of atopic dermatitis: design, validation, and scoring of the Atopic Dermatitis Control Tool (ADCT). Curr Med Res Opin 2020; 36: 367-376.

20. Finlay AY, Khan GK. Dermatology Life Quality Index (DLQI) a simple practical measure for routine clinical use. Clin Exp Dermatol 1994; 19: 210-216.

21. Hongbo $Y$, Thomas CL, Harrison MA, Salek MS, Finlay AY. Translating the science of quality of life into practice: What do dermatology life quality index scores mean? J Invest Dermatol 2005; 125: 659-664.

22. Cohen S, Kamarck T, Mermelstein R. A global measure of perceived stress. J Health Soc Behav 1983; 24: 385.

23. Paradis, Bénédicte 2020-03-03) Comparaison du niveau de stress en fonction du profil social et académique des étudiants rennais en première année de licence,/Université de Rennes 1.

24. Charman CR, Venn AJ, Williams HC. The Patient-Oriented Eczema Measure: development and initial validation of a new tool for measuring atopic eczema severity from the patients' perspective. Arch Dermatol 2004; 140: 1513-1519.

25. Charman CR, Venn AJ, Ravenscroft JC, Williams HC. Translating Patient-Oriented Eczema Measure (POEM) scores into clinical practice by suggesting severity strata derived using anchor-based methods. Br J Dermatol 2013; 169: 1326-1332.

26. Institute of Medicine (US) Council on Health Care Technology; Mosteller F, Falotico-Taylor J, editors. Quality of life and technology assessment: monograph of the Council on Health Care Technology. Washington (DC): National Academies Press (US); 1989. 6, Assessing quality of life: measures and utility. [accessed 1 August 2021] Available from: https://www.ncbi. nlm.nih.gov/books/NBK235120/.

27. Ridd MJ, King AJL, Le Roux E, Waldecker A, Huntley AL. Systematic review of self-management interventions for people with eczema. Br J Dermatol 2017; 177: 719-734.

28. Glazer AM, Rigel DS. Analysis of trends in geographic distribution of US dermatology workforce density. JAMA Dermatol 2017; 153: 472.

29. Parcours des patients insuffisants cardiaques sévères, Arrêté Dirnov -2019/13, Agence régionale de santé IDF. 